\title{
The Comparison of Trust Structure between Urban and Rural Residents in China
}

\author{
Guanqiong Yang, Shuxian Zeng \\ School of Government, Beijing Normal University, Beijing, China \\ Email: zengshuxianbnu@163.com
}

Received 9 May 2016; accepted 28 May 2016; published 31 May 2016

Copyright $@ 2016$ by authors and Scientific Research Publishing Inc.

This work is licensed under the Creative Commons Attribution International License (CC BY). http://creativecommons.org/licenses/by/4.0/

(c) (i) Open Access

\begin{abstract}
Trust is an important form of social capital, it promotes the groups developing positively cooperation with each other, and has a closely relation with social economic development. Based on the data of China General Social Survey (CGSS) 2010, this article attempts to compare the difference of social trust structure between the urban and rural in China through three aspects: general trust, special trust and organizational trust. The result found out that the social networks and institutional environment of urban and rural are different, which leads to the development rate of social trust in the urban and rural is not synchronized, the rural residents' trust level is higher than the city. And it seems that the city is apparently losing its own trust system to regulate people's behavior and values while the whole society has the old and new trust system alternating. It is important to improve social trust of China, and establish a proper trust system for the modern economic development and social life. This article finally puts forward that we can improve institutional norm, popularize higher education and develop social autonomy organization to solve the problem.
\end{abstract}

\section{Keywords}

\section{Social Trust, Particularistic Trust, Organizational Trust, Social Capital}

\section{Introduction}

Trust is not only a moral topic, but also the economic development issue, because that fact that people don't trust each other will make a sharp increase in the transaction costs of cooperation, and hinder the social division of labor, which will affect the healthy development of a regional or national economy in the long term. Research on social trust had been beginning since the 1990s in China, scholars such as Yanjie Bian (1997) [1], Siqing Peng (1999) [2], Feixue Wang \&Toshio Yamagishi (1999) [3], Shaoguang Wang (2003) [4] focus on social capital, 
political trust, interpersonal relationship and social network, which tried to construct social trust structure of China in theory. However, what the majority of the existing studies reach is analyzing the Chinese's trust attitude and behavior characteristics in overall level, or comparing the discrepancy of social trust between China and other countries. There are few studies specifically explaining the difference of trust within the same China society, from the perspective of comparison between urban and rural, two social systems with great difference in socioeconomic development path, life style and cultural tradition and so on. To make up this deficiency, our study will discuss the topic based on the data of China General Social Survey (CGSS) $2010^{1}$, compare the trust structure of urban and rural residents, and find out the related factors, to more truly and specifically reflect the present situation of social trust in China.

\section{Social Trust, Social Network and Institutional Environment}

What is trust? Much has been written about the conceptual of trust from various disciplines perspective. Based on the previous researches, we conclude two mainstream views. On one side, trust is considered as a non-rational human emotion, including cognitive, emotional and behavioral factors, the behavior being pinned will bring positive expectations, increase the willingness to take risks, scholars analyze from the psychological tendency of individual or collective (Qi Yao, Huawei Ma, 2003) [5]. On the other side, trust exists as a rational choice behavior, since it has many advantages just like cutting down the cost of collecting information, supervision and execution, enhancing the flexibility of the transaction, which is considered as an important way to reduce the transaction cost by economist (Sabel, 1993) [6]. No matter as a non-rational human emotion or as a rational choice behavior, trust is a kind of collective consciousness or value, regulates members' behaviors only in a certain range of social groups. Trust is also though as the crucial component of social capital in present studies, some scholars like Francis Fukuyama, Hongyun Zhou even equivalent it to the social capital, because trust is one of the important emotions people use to maintain their social intercourse, like a big machine can't operate without lubricating oil. Elinor Ostrom's research that social capital like reciprocity, norms, networks and family form the trust, which can restrict the opportunism behavior effectively, enhance the efficiency of collective action and promote voluntary organizations cooperation, it is an effective way to solve the collective action dilemma (Hongyun Zhou, 2011) [7].

Social network is constructed by some similar characteristics such as blood, geopolitical, occupation and interests together to reflect people's social relationship in communication. And the dimensions of scale, density, homogeneity, heterogeneity, cohesion and closure properties affect the way people go to interaction and exchange. The open and inclusive social network can make different groups to establish contact, while groups have strong and exclusive heterogeneous correlation in the closed and dense social network, there are weak contact between groups (Hongyun Zhou, 2011) [7]. Yanjie Bian (1997) [1], Jiade Luo \& Yongzhu Ye (2007) [8], Shaoguang Wang \& XinLiu (2002) [4] believe that Chinese trust relationship is in accordance with the difference sequence pattern in the form of distribution, kinship family deserved more trust than friends, and friends deserved more trust than the acquaintances and strangers. Some researches focus on the network of China's urban residents, they believe that daily social network of urban residents can be divided into three kinds of trust, namely universal trust, general trust and particularistic trust (Weimin Li \&Yucheng Liang, 2002 [9]; Rong Hu \& Jingya Li, 2004 [10]). Rong Hu (2005) [11] research that the social interaction of China rural residents generally include relatives exchanges and village folk exchanges, compare with city, fewer population mobility happen in rural areas, so there are easier for them to keep a relatively stable relationship and people are more unity, the increase in external population will not be conductive to the establishment of the trust of rural areas. The differential sequence of psychological and emotional intimacy between each other determines the relationship and trust level, as the Chinese proverb says, better good neighbours near than relations far away, but due to lack of emotional connotation, trust between friends, acquaintances and random strangers is uncertain and easy to change, this kind of trust is generally short (Weimin Li, Yucheng Liang, 2002) [9].

Social norms and institutions, in essence, is the social and cultural rules provide a system of mutual supervision for the production, exchange and distribution process. There are two kinds of views discussing how norms or institutions affect trust in the previous studies. One is the cultural determinism, cultural norms and values play

\footnotetext{
${ }^{1}$ Data analyzed in this paper were collected by the research project "Chinese General Social Survey (CGSS)" carried out by the National Survey Research Center, Renmin University of China (NSRC). The authors appreciate the assistance in providing data by the institutes and individuals aforementioned. The views expressed herein are the authors' own.
} 
important role on the formation of trust, it can promote or inhibit the development progress, affect the principal exposure to risk. What's more, cultural tradition has the influence on trust in the process of social intergenerational transmission and social environment changed (Patricia M. Doney, Joseph P. Cannon, 1998 [11]; Peter Thisted Dinesen, 2012 [13]). The other research perspective is studying the effect of democratic institutions, laws, regulations and market economic system on social trust. In some institutional environment, trust can be enhanced while the other system environment is not conducive to the development of trust. Game theory is a typical example that it illustrates how the institution environment affects trust. Game theory holds that trust is generated in individual repeated game behaviors, tit for tat strategy will lead to cooperation results for both participants in the game. Weiying Zhang \& Rongzhu Ke (2002) [14] believe that trust and economic performance play promotion role in the society. Trust level is much higher in the developed regions because the frequency of repeated game is higher and transactions mechanism is developed better, so low transaction costs is need to reach faster economic growth, likewise, the development of market economy also contribute to social trust.

The formation and development of trust are formed and developed in a certain social network and institutional environment. Chinese society has a relative abundance of traditional social capital, especially in rural areas, but lack of modern social capital. There will be a long period of time that social capital exists with diverse characteristics simultaneously (Xiaodong Huang, 2011) [15]. And this will be must lead to diverse coexistence pattern of social trust. Since the 1949, China's urban and rural have run in its own development path, the economic level, production distribution and government policy support are different. Modern life style and urbanization have different degrees of shocks on traditional culture system, which lead to the urban and rural have its own social network and institutional environment. Especially when the gap of economic development in urban and rural areas is further widening, the social structure is also different. Based on the effect social network and institutional environment on social trust, our study tries to explore two questions, first, studying the correlation between urban and rural social network and residents' trust level, second, discussing the correlation between urban and rural institutional environment and residents' trust level.

\section{Data and Variables}

The data in the study comes from the China General Social Survey (CGSS) 2010, the survey has 11,783 valid samples, involving residents in thirty-one provinces, autonomous regions and direct Municipality, 7222 samples of urban residents and 4561 samples of rural residents. The survey contains respondents' demographic characteristics such as regional, provincial, residence, gender, education, political affiliation, income. The samples of women accounts for $51.8 \%$, the male is $48.2 \%$, in the political affiliation, Communist Party members accounted for $12.4 \%$, non party membership is $87.6 \%$. There are $13 \%$ respondents never received any education, primary accounted for $22.1 \%$, 29.3\% junior middle school, $12.8 \%$ high school(vocational high school and ordinary high school), $15.4 \%$ college and undergraduate degree or above. In this study, we select variable of individual income, education, political affiliation, gender to analyze the residents' trust level. Economic income and education are considered to change individual's social attitude and enhance the ability of defending social risks, the key factors promote social mobility and hierarchical. The identity of the Chinese Communist Party in China is considered to be a symbol of power and social status.

Based on previous studies of Yamagishi Toshio (1994) [16] and Weimin Li \& Yucheng Liang (2002) [9], we divide the trust into general trust, particularistic trust and organizational trust. The variable of general trust measures current social trust level by directly asking respondents' trust attitude to other people in the society. Questionnaire design the question like "Generally do you agree with that most people in the society are can be trusted", "Do you agree with that others will think of taking advantage of you if you are careless". The attitude is divided into five dimensions, include completely disagree, disagree, neutral, agree, strongly agree (completely disagree $=1$, strongly agree $=5$ ). Since social network is composed of groups with some similar characteristics, we can compare the structure of urban and rural social network through analyzing the particularistic trust of residents. Particularistic trust is measured by asking residents' attitude to the special groups, including family members, relatives, friends, colleagues, superiors, businessmen, schoolmates, fellow-townsmen, religious people, and the trust is divided into five dimensions as the general trust. To analyze effect of institutional environment on social trust, we can measure it through comparing the respondents' organizational trust to all kinds of social organizations. Respondents will be asked the trust attitude to the courts and judicial system, the central and local governments, army, public security department, media, non-government organizations, companies, the National 
People's Congress, religious organizations and schools, each kind of trust also has five subscales. The result of trust attitude in the questionnaire is categorical variables, so we transform the categorical variables into numeric variables, the transformed results will distribute from 0 to 10 , the score is higher the credibility is higher, and the formula as follows:

$$
\text { Scaled response }=50 / 4-(\mathrm{Un}-\text { scaled response }) * 10 / 4
$$

\section{Results and Analysis}

1) Comparison of urban and rural residents' general trust

We use Table 1 to explain something. In the study, we measure general trust level through the question, "Generally do you agree with that most people in the society are can be trusted". As the result shows that the average value of general trust is 6.26, the level of trust attitude is between neutral and agree, urban residents' trust is 6.04, when the rural residents is 6.61. Cautious attitude is measure through the question, "Do you agree with that others will think of taking advantage of you if you are careless", to reflect people's cautious attitude to others, the level belong to neutral. The result shows that Chinese residents don't think them need to maintain a high degree of vigilance at anytime and any occasion when deal with others. The rural residents' cautious attitude is $\mathbf{5 . 1 7}$, while the urban is 4.96 , the difference is not large. It shows that Chinese society is not a low trust society, although people keep a degree of vigilance to others especially stranger, they think they can go to trust others, and the rural residents' general trust level is significantly higher that the urban.

According to Model 1, the Chinese' general trust levels have association with individual economic income and education degree. In the regression equation, individual income has significant statistical significance to the general trust, the regression coefficient is -0.01 , it shows that people with higher income tend to have lower trust level. On education factors, the group who have education only or less than primary school as the benchmark set, we can see the factor of education has a strong statistical significance on general trust. The effect of education shows aU-shape, group of primary school or less has the highest trust level in the investigation, the general trust of group of high school and technical secondary school is the least, and its regression coefficient is -0.639 , the regression coefficient of group of college degree or more is -0.345 . People received college education or more seem to more like to trust others, it suggests to strengthen the education degree of popularization has a positive impact on social trust.

Based on the Model 1, we join the variables of urban and rural into the regression equation, to form a new regression equation, Model 2 analyzes how the difference between urban and rural will impact general trust level. The data shows that the difference of urban and rural is highly correlated with general trust level in China, put the group of rural residents as a benchmark, the general trust of urban residents is fewer 0.486 per unit than rural area. It proves that people live in rural usually more like to trust others than live in urban. In previous studies, economy and education can enhance the risk affordability of individual in the society, so people will more dare to trust strangers, create the opportunities for cooperation and bear the losses caused by risky behavior. But in China, these two factors make people realize the social transaction mechanism is not perfect enough to protect their interest in the risk society, so they avoid to choosing risky behavior and trusting others easily. And this result the urban residents have less intention to trust others than the rural.

Table 1. The regression of general trust.
\begin{tabular}{ccc}
\hline & \multicolumn{1}{c|}{ Model 1 } & Model 2 \\
\hline Individual annual income & $-0.010(-3.202)^{* * *}$ & $-0.009(-2.794)^{* *}$ \\
Junior high school & $-0.440(-7.048)^{* *}$ & $-0.341(-5.375)^{* *}$ \\
High school or Technical Secondary School & $-0.639(-8.993)^{* * *}$ & $-0.427(-5.685)^{* *}$ \\
College or University graduate & $-0.345(-3.202)^{* * *}$ & $-0.071(-0.850)$ \\
Urban \& rural & & $-0.486(-8.480)^{* * *}$ \\
Constant term & $6.585(157.153)^{* * *}$ & $6.768(143.955)^{* *}$
\end{tabular}

Note: 1 . the values in brackets are T test, ${ }^{* *} P<0.01,{ }^{*} P<0.1 ; 2$. Reference group: variable of education is the group of primary school or less, variable of urban\& rural areas is the group of rural areas. 
2) The social network and particular trust of urban and rural residents

Figure 1 shows the average value of particular trust of urban residents and rural residents. Emotional relationship is the standard for the distribution of residents' social intercourse, and it basically in accordance with the current situation as a nepotism society. People still put the family in the most important place in their daily intercourse, family members, relatives, and friends with close relationship deserve the most worthy of trust. But the trust is not limited to special circle, composed by blood relationship and close emotional relationship, for the non-blood relationship groups such as colleagues, superiors, schoolmates and fellow-townsmen, there exist a certain degree of trust. Compare the mean of particular trust of urban and rural, the residents' trust preference are not totally consistent. In the city, the trust level for schoolmates is after family members, relatives and friends, then it's the turn to colleagues and fellow-townsmen, the trust level to businessmen is the lowest. In the rural, fellow-townsmen is after the family members, relatives and friends, then the schoolmates, colleagues, the last group is religious people.

We put the average of all kind of interpersonal trust into rotation factor analysis, get two factors and classified them as affective trust and cognitive trust, the former including family members, relatives and friends, based on kinship or close emotional, these usually have high trustworthiness, the latter are the group mainly have cooperative relations, based on the role cognition in daily life, the trust attitude level is between agree and neutral. According to Table 2, economic income has a significant impact on these two types of trust in the urban sample,

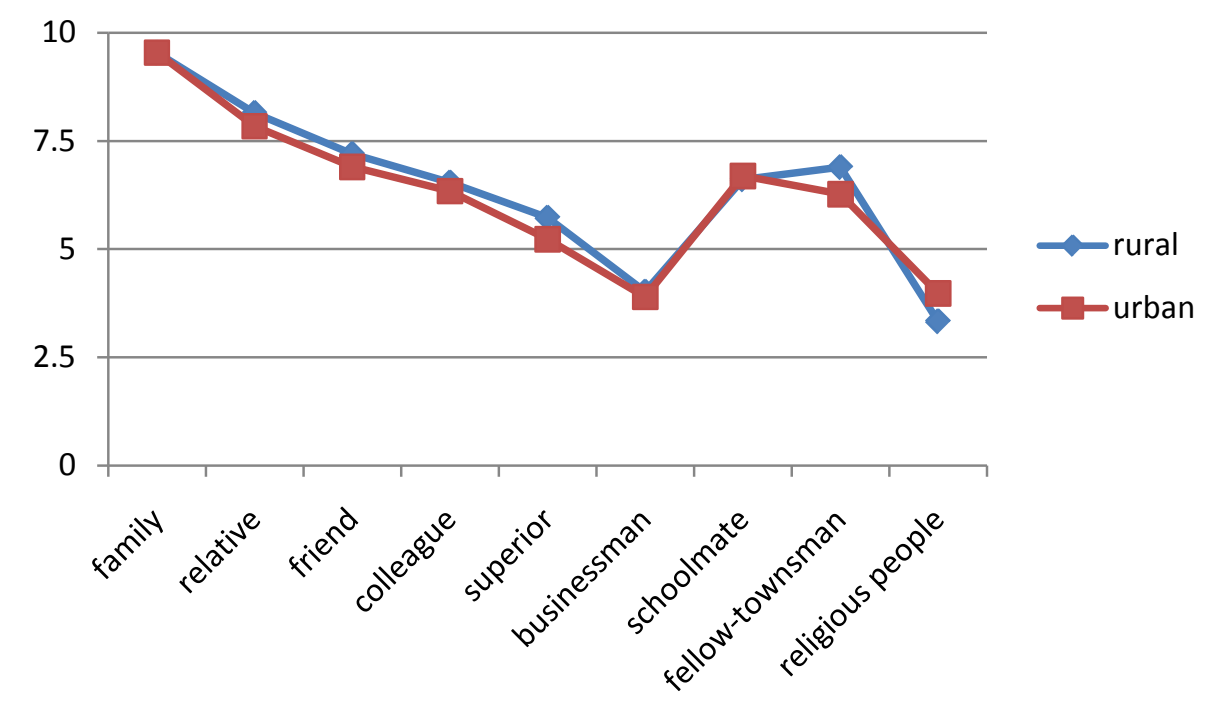

Figure 1. The average of particular trust. Note: the value is from 0 to 10 , the score is higher, the credibility is higher.

Table 2. The regression of particular trust.

\begin{tabular}{ccccc}
\hline & \multicolumn{2}{c}{ Urban } & \multicolumn{2}{c}{ Rural } \\
\cline { 2 - 5 } & affective trust & cognitive trust & affective trust & cognitive trust \\
\cline { 2 - 5 } & Model 3 & Model 4 & Model 5 & Model 6 \\
\hline Individual annual income & $-0.005(-3.065)^{* *}$ & $-0.003(-1.994)^{* *}$ & $0.004(0.503)$ & $-0.005(-0.483)$ \\
Junior high school & $0.028(0.663)^{*}$ & $-0.117(-2.451)^{* * *}$ & $-0.071(-1.569)$ & $-0.137(-2.361)^{* *}$ \\
High school or Technical Secondary School & $0.013(0.311)$ & $-0.103(-2.122)^{* *}$ & $-0.194(-2.614)^{* *}$ & $-0.168(-1.797)^{*}$ \\
College or University graduate & $0.167(3.70)^{* *}$ & $0.111(2.155)^{* *}$ & $-0.039(-0.257)$ & $-0.004(0.022)$ \\
Gender & $0.061(2.032)^{* *}$ & $-0.035(-1.020)$ & $0.059(1.406)$ & $0.088(1.644)^{*}$ \\
Political Affiliation & $0.099(2.355)^{* *}$ & $0.160(3.354)^{* *}$ & $0.194(2.144)^{* *}$ & $0.313(2.753)^{* *}$ \\
Constant term & $8.079(244.970)^{* *}$ & $5.402(144.087)^{* *}$ & $8.302(260.136)^{* *}$ & $5.862(140.281)^{* *}$ \\
\hline
\end{tabular}

Note: 1 . the values in brackets are T test, ${ }^{* *} \mathrm{P}<0.01,{ }^{*} \mathrm{P}<0.1 ; 2$. Reference group: variable of education is the group of primary school or less, variable of gender is woman, variable of political affiliation is the group of non-party relationship. 
the personal income is higher, the level of particular trust attitude is lower, but the impact of income variable on rural model is not significant. The variable of education has statistical significance on cognitive trust in the model of urban, and the influence to trust level is U-shape, group of primary school or less as the benchmark, group of junior high school has the lowest trust level, when people receive education of high school degree or more, trust tend to increase. In the countryside, groups receive less education seem to have high cognitive trust. Variable of political affiliation has significant influence on affective trust and cognitive trust both in the urban and rural. Party members have higher particular trust level than non-party people.

In the study, education has more influence on the cognitive trust than on the affective trust, and the influence is more significantly in urban than in rural. People live in city usually have higher education degree, different from the kinship and geopolitical relationship, schoolmates become the important resource in social interaction, so the social network of city is more inclusive and open. But in rural geopolitical relationship is more preferred in the social intercourse, so social network of rural is relatively closed and dense. In addition, the residents' trust attitude to religious people can also reflect the difference of social network. Lawrence Hrrison believes that every group which exists social capital has its own radius of trust, and cooperation norms are enforced in the circle (Harrison Laurence, 1985) [17]. Trust radius is larger than the scope of group itself can produce a positive external effect, such as religious, advocate trust and responsibility and penetrate the influence to the whole society. In China, people know less about the religious culture, lack of correct religious consciousness in the daily life, so they don't have a good impression of religious people. But the urban residents have higher trust level to the religious people than the rural areas, it indicates that city's social network is more open and inclusive than the countryside.

3) The institutional environment and organization trust of urban and rural residents

Figure 2 shows the trust average level of all kinds of organizations. People have a high recognition to authoritative organization such as the government department, while show low credibility to the non-governmental organizations, companies, and religious organizations. When people choose to trust the government organizations, they believe that central government agencies and organization are more worthy of trust, such as the central government, army, national media and the National People's Congress, by contrast, the credibility of local government agencies and grass-roots organizations are low in society. We can see that people trust more the central government than local government, and also the national media is more preferential than local media. The central government has strong ability to control resources, they have obtained great economic achievements of the economy, public policy and social welfare since 1978, so an authoritative and impartial image have been build up for voters and they win more political support. The local government plays the role of policy transmission, executor and public service providers, is closely related to voters' daily life, the administrative omission

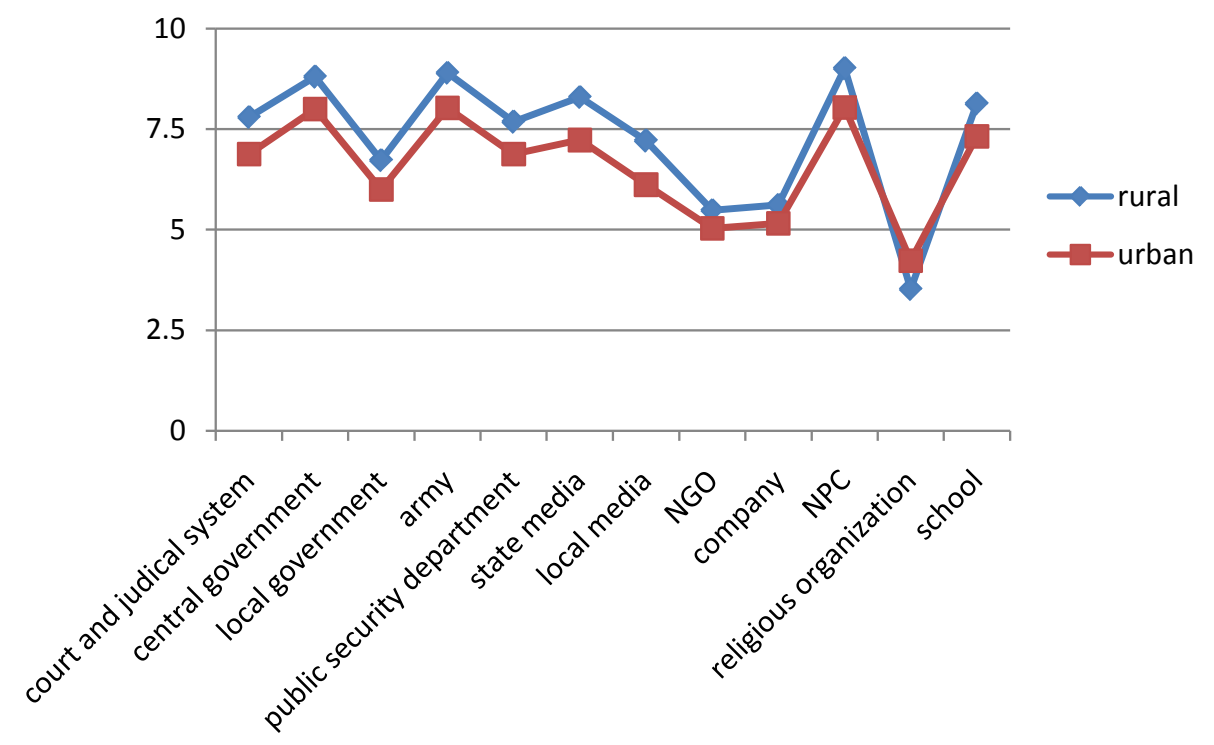

Figure 2. The average of organizational trust. Note: the value is from 0 to 10 , the score is higher, the credibility is higher. 
and irresponsibility may lead to negative image of local officials and influence voters' evaluation. It's easy to cause the loss of political trust.

We study the various organizations and institutions with average rotation factor analysis, as Table 3 shows, take out two factors, the first one is authority organization, including the government department, the NPC, army, media, public security, the courts and judicial system and education system, such kind of organizations and institutions have the characteristics of political consciousness and authority, classified as authoritative trust. Organizations of the second factor have characteristics of independence, justice and autonomy, including nongovernment organizations, companies, and religious organizations, we name the second kind of trust as the autonomous trust.

Table 3 shows that the influences of education on these two kinds of organizational trust are different. Education has statistical significance to the authoritative trust, people received higher degree of education will trust less about the organization. Education of high school degree or less is negatively correlated with the trust, but group of college degree or above has no statistical significance. On the variable of political affiliation, group of communist party members' trust in the authoritative organization is per unit 0.321 higher than non-party members, but it's not statistically significant in the autonomy organizations. Difference between urban and rural areas also have statistical significance on trust level of authoritative organization, the trust of rural residents to authoritative organization is per unit 0.412 higher than urban residents, similarly, there is not any statistical significance in the organizations spontaneously.

China has been at the same structure of clan and country for a long period. Government regulates and controls social resources configuration, deeply involves in citizens' life and the development of social organizations, set up a strong authoritative trust. This is more obvious in the countryside, national-oriented and clan-oriented still dominate the rural residents' trust model. However, with the enhancement of economic income and improvement of education level, people especially in the towns and cities are not easily satisfied with the governments' public services. Authoritative trust has been questioned. In addition, both in rural and urban, the Chinese people's trust of social autonomy organizations is low. People have low trust level to companies, it can also be proved from the perspective that economic income level is inversely proportional with the trust level and people's confidence in the business is at the low degree. The main reason is that market trading rules, especially the supervision and sanctions regime are imperfect, market transactions make every participants face with a great deal of risk. At the other side, intellectual property protection mechanism is weak, citizens' property can't get lawful protection, so in China high-earning people have much more cautious and alert to the society. The development scale of non-governmental organizations is small and not enough mature, people don't have much opportunities to participate in activities of such kind organizations, so they distrust autonomy organizations like non-governmental organizations and religious organizations. Thus non-governmental organization don't play a key role in the management of social public affairs, the social resources have not been effectively utilize.

Table 3. The regression of organizational trust.

\begin{tabular}{ccc}
\hline & authoritative trust & autonomous trust \\
\cline { 2 - 3 } Individual annual income & Model 7 & Model 8 \\
Junior high school & $-0.011(-5.822)^{* *}$ & $-0.004(-1.988)^{* *}$ \\
High school or Technical Secondary School & $-0.426(-11.108)^{* * *}$ & $-0.192(-4.391)^{* *}$ \\
College or University graduate & $-0.661(-14.456)^{* * *}$ & $-0.223(-4.275)^{* *}$ \\
Political Affiliation & $-0.997(-18.971)^{* *}$ & $0.079(1.311)$ \\
Urban\& rural & $0.321(0.062)^{* *}$ & $0.008(0.144)$ \\
Constant term & $-0.412(-0.117)^{* *}$ & $0.023(0.569)$ \\
\hline
\end{tabular}

Note: 1 . the values in brackets are T test, ${ }^{* *} P<0.01,{ }^{*} P<0.1 ; 2$. Reference group: variable of education is the group of primary school or less, variable of urban\& rural areas is the group of rural areas, variable of political affiliation is the group of non-party relationship. 


\section{Conclusion and Discussion}

The trust social network in China is gradually becoming open and inclusive, people not only confined social interaction in the blood relationship circle, most people think others in society are deserved to believe. Cognitive trust has become an important part of social capital. However, at the background of increasingly diversified social environment and complicated interest structure, old trust system dominated by traditional culture is gradually weakening its influence of regulation, it' s much apparently in the city, while the impact on rural is relatively weak, and still retained the most of the traditional trust model. The old trust system is gradually collapsed, but the new trust system has not yet been established. Legal system is incomplete, administrative rules and regulations are fragmented, and lack of binding norms and effective rules in the society easily causes social disorder. People feel confused and uncertainty, so they often tend to be more conservative on the selection strategy when choosing the cooperative partner, or through acquaintances preferences to select, resulting in a decline in social trust level. It also explains why in the rapid economic development of cities and towns, social networks are more open and inclusive, but residents' expectations of social trust are lower than the countryside.

To reshape a new trust system to adapt to urbanization and development of market economy, firstly, government should perfect social norms to keep social order steady and certain, change the status quo of fragmented legal system, optional explanation as well as mutual conflict of rules, regulations, administrative regulations. At the same time, it should establish rewards and punishments mechanism, create a favorable environment for trading cooperation climate of trust, and make both parties acting in the form of trust. Secondly, promoting the popularization of higher education, it's significant for improving the quality of the members of the organization to increase social trust, since the result shows that education has a U-shaped influence to the social trust, group of receiving higher education is more likely to trust others. And lastly, rebuilding trust needs to encourage citizens to actively participate in activities of voluntary associations, improve the social participation to promote the development of civil social organizations, making it a third mechanism outside of government and market to solve the social problems and deal with public affairs. It will expand the richness of social capital.

\section{Fund}

This work was supported in part by the National Social Science Foundation of China (Grant No. 13AZD093 and 15BGL154). The views expressed herein are those of the authors and are not necessarily those of the National Social Science Foundation of China.

\section{References}

[1] Bian, Y.J. (1997) Guanxi Networks and Job Mobility in China and Singapore. Social Forces, 75, 981-1005. http://dx.doi.org/10.1093/sf/75.3.981

[2] Yang, Z.F. and Peng, S.Q. (1999) Conceptualization of Interpersonal Trust in China: A Perspective of Interpersonal Relationship. Sociological Study, No. 2, 3-23.

[3] Wang, F.X. and Toshio,Y. (1999) A Comparative Study of Trust in China, Japan and America. Sociological Study, No. 2, 69-84.

[4] Wang, S.G. and Liu, X. (2002) The Foundation of Trust: A rational Explanation. Sociological Study, No. 3, 23-39.

[5] Ma, H.W. and Yao, Q. (2013) Contemporary Trust Research From the Perspective of Social Psychology. China Legal Publishing House, Beijing.

[6] Sabel, C.F. (1993) Studies Trust: Building New Forms of Cooperation in a Volatile Economy. Human Relations, 46, 1132-1170.

[7] Zhou, H.Y. (2011) Social Capital and Democracy. Social Science Academic Press, Beijing.

[8] Luo, J.D. and Ye, Y.Z. (2007) Chinese’s Trust Game. Social Science Academic Press, Beijing.

[9] Li, W.M. and Liang, Y.C. (2002) Particular Trust and General Trust: the Structure and Characteristics of Chinese Trust. Sociological Study, No. 3, 11-22.

[10] Hu, R. and Li, J.Y. (2006) The Composition and Influencing Factors of Urban Residents’ Trust. Chinese Journal of Sociology, 26, 45-61.

[11] Hu, R. (2005) Social Trust of Rural Residents in China. Journal of the Party School of Tianjin Committee of the CPC, No. 2, 46-51.

[12] Doney, P.M. and Cannon, J.P. (1998) Understanding the Influence of National Culture on the Development of Trust. 
The Academy of Management Review, 23, 601-620.

[13] Dinesen, P.T. (2012) Does Generalized (Dis)Trust Travel? Examining the Impact of Cultural Heritage and Destination-Country Environment on Trust of Immigrants. Political Psychology, 33, 495-511. http://dx.doi.org/10.1111/j.1467-9221.2012.00886.x

[14] Zhang, W.Y. and Ke, R.Z. (2002) Trust in China: A Cross-Regional Analysis. Economic Research Journal, No. 10, 59-70.

[15] Huang, X.D. (2011) Social Capital and Government Governance. Social Science Academic Press, Beijing.

[16] Toshio, Y. (1994) Trust and Commitment in the United States and Japan. Motivation and Emotion, 18, 129-166. http://dx.doi.org/10.1007/BF02249397

[17] Harrison, L. (1985) Under Development Is a State of Mind: The Latin American Case. Madison Books, New York. 